\title{
Analyse des modes de défaillance, de leurs effets et de leur criticité dans le circuit du médicament : revue de littérature
}

\author{
Émile Demers, Laurence Collin-Lévesque, Marianne Boulé, Sophie Lachapelle, Christina Nguyen, \\ Denis Lebel et Jean-François Bussières
}

\section{RESUMÉ}

Contexte : L'analyse des modes de défaillance, de leurs effets et de leur criticité (AMDEC) est une méthode d'analyse systématique et proactive des risques permettant de déterminer les défaillances majeures de processus complexes.

Objectif : Recenser tous les articles concernant l'utilisation de l'analyse des modes de défaillance et de leurs effets (AMDE), de l'AMDEC et de l'AMDEC en santé (AMDECS) dans le cadre du circuit du médicament.

Sources des données, sélection des études et extraction des données : La base de données MEDLINE a été interrogée pour la période de janvier 1990 à janvier 2017. La stratégie de recherche a inclus les études appliquant intégralement ou partiellement la méthode AMDEC et traitant d'un ou de plusieurs volets du circuit du médicament. Une recherche manuelle complémentaire a permis d'inclure les références pertinentes des articles consultés.

Synthèse des données : Les chercheurs ont trouvé 171 articles. Ils en ont retenu 39, soit 32 décrivant l'utilisation de l'approche AMDE ou AMDEC et sept décrivant l'utilisation de l'approche AMDECS. Ils ont répertorié de quatre à 378 modes de défaillance, selon les études publiées. Dix des 39 articles font état d'une analyse avant et après la mise en vigueur de mesures correctives. Dans quatre de ces 10 articles, l'analyse a été réalisée de façon théorique, soit avant la mise en vigueur réelle des mesures. À partir des articles retenus, un tableau-synthèse a été élaboré avec les éléments suivants : année de publication, premier auteur, pays, objectif principal, objectifs secondaires, description de la méthode, description des résultats, commentaires. Le tableau-synthèse a permis de commenter l'état d'utilisation des analyses de type AMDEC dans le cadre du circuit du médicament.

Conclusions : Cette revue de la littérature a retenu 39 articles publiés ayant utilisé l'approche AMDE, AMDEC ou AMDECS dans le cadre du circuit du médicament. La plupart des études ont utilisé l'approche AMDE ou AMDEC, tandis que l'AMDECS n'était que rarement employée. Seule une minorité des études ont évalué les effets de mesures correctives mises en œuvre. Cette approche permet la cartographie d'un processus de soins, la détermination des modes de défaillance et la priorisation des actions correctives. Il faudrait encourager son usage pour l'évaluation du circuit du médicament.

Mots-clés : analyse des modes de défaillance et de leurs effets; analyse des modes de défaillance, de leurs effets et de leur criticité; analyse des modes de défaillance, de leurs effets et de leur criticité en santé; médicament; mode de défaillance; criticité; risques

J. Can. Pharm. Hosp. 2018;71(6):376-84

\begin{abstract}
Background: Failure mode, effects, and criticality analysis (FMECA) is a systematic and proactive risk analysis method to determine major failures in complex processes.

Objective: To identify all articles involving the use of failure mode and effects analysis (FMEA), FMECA, or FMECA in health care within the medication use system.
\end{abstract}

Data Sources, Study Selection, and Data Extraction: The MEDLINE database was searched, for the period January 1990 to January 2017. The search included studies using the FMECA method, in part or in full, and dealing with one or several components of the medication use system. The reference lists of articles identified in the initial search were checked manually for additional pertinent references.

Data Synthesis: The researchers identified 171 articles, and retained 39 for analysis: 32 describing use of the FMEA or FMECA approach and 7 describing use of the FMECA in health care approach. They identified between 4 to 378 failure modes, according to the published studies. Among the 39 articles, 10 reported a pre- and post-implementation analysis of corrective measures. In 4 of those 10 articles, the analysis was conducted on a theoretical basis, that is, before the corrective measures were actually implemented. Using the articles retained for analysis, a summary table was developed with the following elements: publication year, main author, country, primary objective, secondary objectives, descriptions of both method and results, and comments. The summary table gave the opportunity to comment on the use of the FMECA-type analysis within the medication use system.

Conclusions: This literature review included 39 published articles using an FMEA, FMECA, or FMECA in health care approach within the medication use system. Most studies used either the FMEA or the FMECA approach, whereas the FMECA in health care approach was used only rarely. Only a minority of studies assessed the effects of corrective measures that were implemented. This overall approach allows for mapping of a care process, determination of failure modes, and prioritization of corrective measures. Its use for the assessment of the medication use system should be promoted.

Keywords: failure mode and effects analysis; failure mode, effects, and criticality analysis; failure mode, effects, and criticality analysis in health care; drug; failure mode; criticality; risks 


\section{CONTEXTE}

$\mathrm{L}^{\prime}$ analyse des modes de défaillance, de leurs effets et de leur criticité (AMDEC) est une méthode d'analyse systématique et proactive des risques permettant de déceler les défaillances majeures de processus complexes ${ }^{1-3}$. L'AMDEC pose comme axiome que l'organisation même d'un système, et non les individus qui le composent, doit être au cœur de toute démarche de réduction des risques ${ }^{3}$. De là, l'idée d'une erreur associée à un individu est remplacée par l'idée d'un mode de défaillance associé à un système.

Une AMDEC est un processus multidisciplinaire qui permet de coter trois indices et d'en calculer le produit par mode de défaillance ainsi que la somme de ces produits ${ }^{3}$. De façon générale, une échelle de cotation numérique sert à calculer chaque indice, soit un indice de fréquence de survenue de chaque mode de défaillance, un indice de sévérité des conséquences de la survenue du mode de défaillance et un indice de capacité de détection du mode de défaillance. Ainsi, pour un mode de défaillance donné et coté à l'aide d'échelles de 1 à 10 , le risque le moins élevé donne une valeur de $1(1 \times 1 \times 1)$ alors que le risque le plus élevé donne un produit de $1000(10 \times 10 \times 10)$, ce risque est nommé l'indice de criticité (IC) dans la littérature. À noter que l'analyse des modes de défaillance et de leurs effets (AMDE) est une analyse similaire sans évaluation de la criticité.

L'AMDEC a été créée par l'armée américaine dans les années 1950 et a par la suite été popularisée par la NASA, les constructeurs aéronautiques et l'industrie automobile ${ }^{4-6}$. L'AMDEC a fait son apparition dans le domaine de la santé dans les années $1990^{7}$.

Aux États-Unis, The Joint Commission a publié des principes méthodologiques directeurs relatifs à l'AMDEC ${ }^{8,9}$. La démarche proposée consiste en la sélection d'un processus comportant un risque élevé, la constitution d'une équipe multidisciplinaire d'experts, la schématisation exhaustive des étapes du processus à l'étude, la détermination par remue-méninges de tous les modes de défaillance possibles, la détection des causes et des effets de chaque mode de défaillance, la hiérarchisation des modes de défaillances, l'implantation de mesures correctives, l'analyse du nouveau processus et l'évaluation des changements accomplis.

Au début des années 2000, le Veterans Administration National Center for Patient Safety a développé une variante de l'AMDEC, soit l'AMDEC en santé (AMDECS aussi appelé Healthcare failure mode effect analysis ${ }^{10}$. The Joint Commission estime que l'AMDECS est plus facile à appliquer en santé en raison de la simplification et de l'amélioration de la démarche. LAMDECS comprend notamment des échelles de cotations simplifiées (p. ex. score de 1 à 4 plutôt que de 1 à 10) et exige une prise de décision relative à chaque mode de défaillance détecté : " accepter ", " contrôler " ou "éliminer ". Une personne responsable est nommée pour assurer le suivi de chaque mode de défaillance à contrôler et à éliminer. Néanmoins, la méthode d'analyse des risques demeure identique à celle de l'AMDE / AMDEC.
Pour les professionnels travaillant en établissement de santé, les AMDE / AMDEC / AMDECS représentent des approches utiles qui peuvent contribuer à améliorer les pratiques et qui satisfont à au moins une pratique organisationnelle requise d'Agrément Canada (c.-à-d. la tenue d'analyses prospectives liées à la sécurité des usagers ${ }^{11}$. Nous ignorons dans quelle mesure ils connaissent l'approche AMDEC et s'ils ont accès aux articles ayant recours à cette approche.

Recenser tous les articles concernant l'utilisation de l'approche AMDE / AMDEC / AMDECS dans le cadre du circuit du médicament.

\section{SOURCES DES DONNÉES, SÉLECTION DES ÉTUDES ET EXTRACTION DES DONNÉES}

La méthodologie repose sur l'interrogation de la base de données MEDLINE pour la période de janvier 1990 à janvier 2017 à l'aide de la stratégie suivante : " Healthcare Failure Mode and Effect Analysis"[mh] OR "Healthcare Failure Mode and Effect Analysis" [all] OR "Failure Mode and Effect Analysis" [all] OR "FMEA"[all] OR "FMECA"[all]) AND ("Drugs" [all] OR "Medication"[all] OR "Hospital"[all] ) AND ("1990/01/01" [pdat] : "2017/01/27”[pdat] ». Les investigateurs ont en outre effectué une recherche manuelle complémentaire portant sur les références des articles consultés.

L'un des auteurs (E.D.) a effectué une première sélection des articles à partir du titre. Puis, à partir du résumé structuré, il a procédé à l'inclusion des articles correspondant aux critères d'inclusion suivants : études appliquant intégralement ou partiellement la méthode AMDEC et traitant d'un ou de plusieurs volets du circuit du médicament. Un autre auteur (J.F.B.) a vérifié les raisons de sélection et de refus des résumés structurés. La consultation des autres auteurs a permis de résoudre les divergences. Les AMDEC portant sur d'autres processus, comme la communication entre professionnels de la santé ou l'admission aux urgences, étaient incluses dans la mesure où le circuit du médicament occupait une place prépondérante dans l'énumération des étapes et des modes de défaillance. Les articles traitant uniquement des procédés industriels de fabrication des médicaments ont été exclus, de même que les articles publiés dans une langue autre que l'anglais ou le français.

À partir des articles retenus, nous avons élaboré un tableau synthèse comportant les éléments suivants : année de publication, premier auteur, pays, objectif principal, objectifs secondaires, description de la méthode, description des résultats, commentaires. À partir du tableau synthèse, nous avons commenté l'état de l'utilisation des analyses de type AMDE / AMDEC / AMDECS dans le cadre du circuit du médicament. Nous avons procédé uniquement aux analyses des statistiques descriptives.

\section{RÉSULTATS}

Les chercheurs ont trouvé 171 articles. Ils en ont retenu 39, soit 32 décrivant l'utilisation de l'approche AMDE ou 
$\mathrm{AMDEC}^{5,12-42}$ et sept décrivant l'utilisation de l'approche $\mathrm{AMDECS}^{43-49}$. Plusieurs articles retenus utilisent les termes " $\mathrm{AMDE}$ » et " AMDEC » sans distinction, peu importe la présence ou l'absence d'un calcul de criticité dans leur méthodologie; les résultats présentés ici combinent donc l'ensemble de ces articles. La figure 1 illustre le processus de sélection des articles.

Des 39 études prises en compte, 11 proviennent des ÉtatsUnis, cinq du Canada, cinq d'Espagne, quatre de Suisse et 14 d'autres pays. Presque toutes les études $(n=37)$ portent sur des processus dans les unités de soins ou des cliniques externes, contre 22 dans les départements de pharmacie. Les équipes multidisciplinaires engagées dans la tenue de l'AMDE / AMDEC / AMDECS sont formées de professionnels exerçant entre deux et huit professions, selon les études. Les plus concernés étaient les médecins, les infirmières, les pharmaciens et les gestionnaires. Les participants à ces analyses ont décelé de quatre à 378 modes de défaillance, selon les études publiées.

En ce qui concerne le dépistage des modes de défaillance, il était généralement réalisé par consensus lors d'une rencontre de l'équipe de recherche. La cotation des différents modes de défaillance s'est faite de façon consensuelle $(n=32)$, mais elle correspondait parfois à la moyenne des cotations individuelles des participants $(n=3)$. Pour leur part, Kunac et Reith ${ }^{26}$ ont opté pour une cotation des modes de défaillances par la médiane. Hosoya et collab. ${ }^{24}$ ont utilisé des cotations obtenues par sondage de patients et non par consensus d'experts. Ashley et Armitage ${ }^{43}$ ont eu recours à deux modes de cotation, soit celle par consensus et celle par moyenne. Les résultats obtenus indiquent que les IC obtenus par consensus sont plus élevés que ceux obtenus par moyenne en général. De façon générale, la plupart des IC sont calculés pour chacun des modes de défaillances détectés et pour chacune des étapes du processus. Plusieurs auteurs ont aussi calculé l'IC global du processus à l'étude. Certains auteurs ont modifié légèrement la méthodologie conventionnelle. Par exemple, Apkon et collab. ${ }^{13}$ ont calculé des IC associés à des processus et non aux modes de défaillance ni aux sous-étapes constituant le processus. Armitage et collab. ${ }^{14}$ ont coté indépendamment chacune des causes associées à chaque mode de défaillance, au lieu de coter directement chacun des modes de défaillance.

Enfin, les valeurs des indices de criticité recensées variaient de sept à 729 (c.-à-d. administration du mauvais médicament ou de la mauvaise dose) tandis que dans les AMDECS, les valeurs recensées variaient de huit à 16 . Le tableau 1 présente un profil synthèse des AMDE / AMDEC utilisés dans le circuit du médicament.

Des 39 études prises en compte, 10 d'entre elles sont des analyses de type pré et postimplantation de mesures correctives. Quatre de ces analyses pré et postimplantation se sont déroulées en fait avant l'implantation réelle des mesures correctives; les panélistes étaient invités à évaluer les effets potentiels des mesures correctives avant qu'elles aient été implantées ${ }^{18,19,31,36}$. Par ailleurs, une autre de ces analyses est une sorte d'évaluation pré et postimplantation a posteriori : Bonnabry et collab. ${ }^{17}$ ont réalisé une AMDEC sur un processus avant et après des mesures correctives déjà implantées au moment des séances de remue-méninges. Lorsqu'elles sont explicitées, les mesures correctives varient d'un article à l'autre. Lago et collab. ${ }^{27}$ énoncent une série de mesures entreprises à la suite de leur analyse, entre autres en ce qui a trait à la double vérification, à la création de nouvelles feuilles d'ordonnances prérédigées, à l'implantation du bilan comparatif des médicaments au transfert des patients, à la diminution du nombre de formats multidoses disponibles et à l'utilisation d'étiquettes de couleur pour repérer les dossiers et les préparations de chimiothérapie.

Le tableau 2 présente un profil synthèse des AMDECS utilisés dans le circuit du médicament. Aucune de ces AMDECS ne présentait d'analyse de type pré et postimplantation.

\section{DISCUSSION}

À notre connaissance, il s'agit de la première revue de littérature recensant tous les articles ayant utilisé l'approche AMDE / AMDEC / AMDECS dans le cadre du circuit du médicament de 1990 à 2017. Il s'agit d'une approche utilisée surtout dans les pays anglo-saxons (c.-à-d. États-Unis, RoyaumeUni, Canada, Nouvelle-Zélande) mais également dans la francophonie (France, Suisse, Canada).

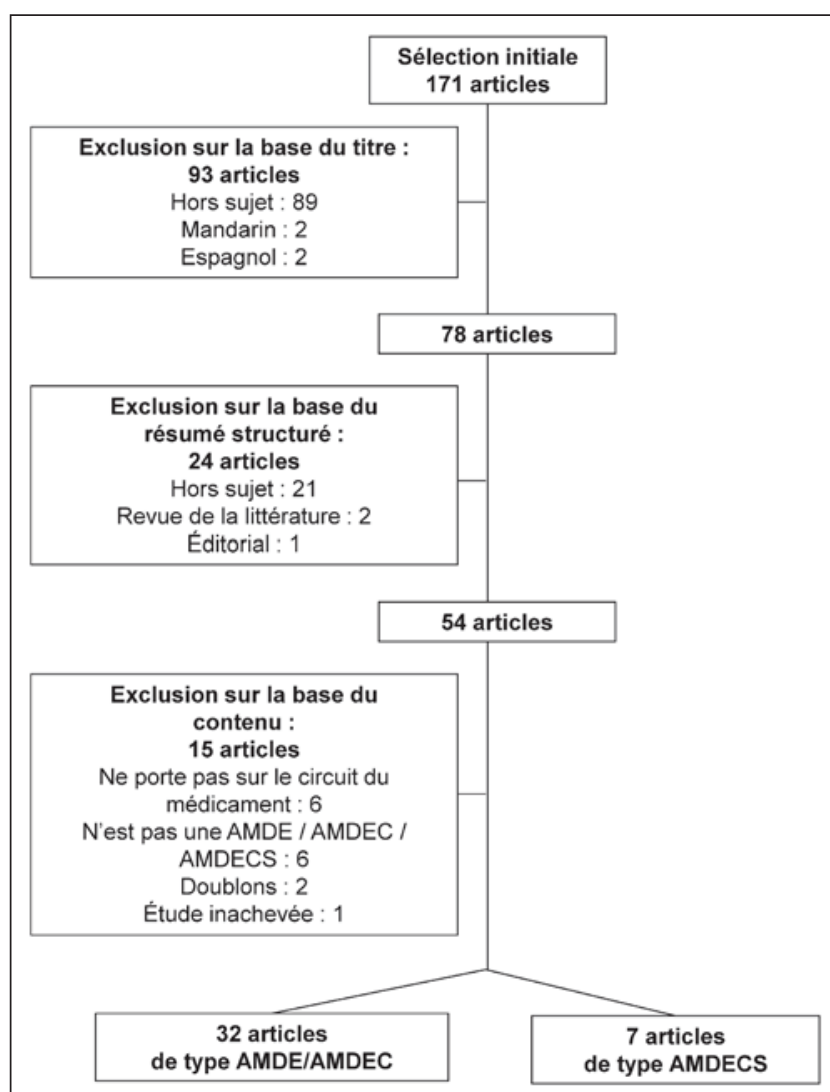

Figure 1. Processus de sélection des articles. 


\section{Tableau 1 (partie 1 de 2). Profil synthèse des AMDE et des AMDEC utilisées dans le circuit du médicament}

\begin{tabular}{|c|c|c|c|c|c|c|}
\hline Référence & Pays & $\begin{array}{l}\text { Processus en } \\
\text { pharmacie (PH) } \\
\text { ou en unités de } \\
\text { soins, de cliniques } \\
\text { externes (UC) }\end{array}$ & $\begin{array}{l}\text { Nombre de } \\
\text { professions } \\
\text { représentées } \\
\text { dans l'équipe } \\
\text { de recherche }\end{array}$ & $\begin{array}{l}\text { Nombre de } \\
\text { modes de } \\
\text { défaillance } \\
\text { décelés }\end{array}$ & $\begin{array}{l}\text { Interventions (protocole } \\
\text { avec AMDE ou AMDEC } \\
\text { pré-post seulement) } \\
\text { et évaluation } \\
\text { post-implantation } \\
\text { (implantation réelle ou } \\
\text { simulée) }\end{array}$ & $\begin{array}{c}\text { Mode de } \\
\text { défaillance } \\
\text { comportant IC } \\
\text { le plus élevé } \\
\text { (valeur de l'IC } \\
\text { rapportée dans } \\
\text { l'étude) }\end{array}$ \\
\hline $\begin{array}{l}\text { Adachi et Lodolce } \\
(2005)^{12}\end{array}$ & États-Unis & $\mathrm{PH}+\mathrm{UC}$ & 4 & 5 & NA & $\begin{array}{l}\text { «Programmer la } \\
\text { pompe »(246) }\end{array}$ \\
\hline Apkon et al. $(2004)^{13}$ & États-Unis & $\mathrm{PH}+\mathrm{UC}$ & 5 & 6 & $\begin{array}{l}\text { Mesures correctives } \\
\text { diverses (réel) }\end{array}$ & $\begin{array}{l}\text { "Programmer la } \\
\text { pompe » (269 pré, } \\
99 \text { post) }\end{array}$ \\
\hline $\begin{array}{l}\text { Armitage et al. } \\
(2011)^{14}\end{array}$ & Swaziland & $\mathrm{PH}$ & 2 & 11 & NA & $\begin{array}{l}\text { « Mauvais } \\
\text { médicament / dose } \\
\text { car personnel non } \\
\text { formé » (729) }\end{array}$ \\
\hline Baker et al. (2008) & États-Unis & UC & 6 & ND & NA & $\begin{array}{l}\text { "Prescriptions } \\
\text { identifiées au mauvais } \\
\text { patient » (420) }\end{array}$ \\
\hline $\begin{array}{l}\text { Berruyer et al. } \\
(2014)^{16}\end{array}$ & Canada & $\mathrm{PH}+\mathrm{UC}$ & 4 & 49 & $\mathrm{NA}$ & $\begin{array}{l}\text { "Non-vérification de } \\
\text { l'identité du } \\
\text { patient » }(600)\end{array}$ \\
\hline $\begin{array}{l}\text { Bonnabry et al. } \\
(2005)^{17}\end{array}$ & Suisse & $\mathrm{PH}$ & 2 & 18 & $\begin{array}{l}\text { Informatisation du circuit } \\
\text { du médicament (réel } \\
\text { a posteriori) }\end{array}$ & $\begin{array}{l}\text { "Erreur de dosage » } \\
\text { (512 pré, } 64 \text { post) }\end{array}$ \\
\hline $\begin{array}{l}\text { Bonnabry et al. } \\
(2006)^{18}\end{array}$ & Suisse & $\mathrm{PH}+\mathrm{UC}$ & 3 & 27 & $\begin{array}{l}\text { Informatisation du circuit } \\
\text { du médicament (simulé) }\end{array}$ & $\begin{array}{l}\text { « Erreur de dose } \\
\text { dans la production } \\
\text { des protocoles » } \\
\text { (432 pré, } 9 \text { post) }\end{array}$ \\
\hline $\begin{array}{l}\text { Bonnabry et al. } \\
(2008)^{19}\end{array}$ & Suisse & UC & 5 & 27 & $\begin{array}{l}\text { Optimisation de la } \\
\text { prescription électronique } \\
\text { (simulée) }\end{array}$ & $\begin{array}{l}\text { "Prescription } \\
\text { ambiguë, illisible ou } \\
\text { incomplète » (392 } \\
\text { pré, } 148 \text { post) }\end{array}$ \\
\hline Coles et al. $(2005)^{20}$ & États-Unis & $\mathrm{PH}+\mathrm{UC}$ & $\mathrm{ND}$ & ND & $\mathrm{NA}$ & ND \\
\hline $\begin{array}{l}\text { de la Riva et al. } \\
(2015)^{21}\end{array}$ & Espagne & $\mathrm{PH}+\mathrm{UC}$ & 2 & 6 & NA & $\begin{array}{l}\text { "Mauvais } \\
\text { médicament: } \\
\text { emballage similaire » } \\
(300)\end{array}$ \\
\hline Delage et al. $(2015)^{22}$ & Canada & UC & 4 & $\begin{array}{l}11 \text { pré, } \\
16 \text { post }\end{array}$ & $\begin{array}{l}\text { Changement des pompes } \\
\text { intelligentes (réel) }\end{array}$ & $\begin{array}{l}\text { «Erreurs de débit » } \\
\text { (280 pré, } 70 \text { post) }\end{array}$ \\
\hline Dräger (2016) $)^{23}$ & Allemagne & UC & ND & 20 & $\mathrm{NA}$ & ND \\
\hline Hosoya et al. $(2015)^{24}$ & Japon & UC & $\mathrm{ND}$ & 4 & NA & $\begin{array}{l}\text { "Oubli par } \\
\text { distraction }(7 \pm 1)\end{array}$ \\
\hline Kaestli et al. $(2014)^{25}$ & Suisse & $\mathrm{PH}+\mathrm{UC}$ & 5 & 23 & NA & $\begin{array}{l}\text { "Mauvaise dose de } \\
\text { morphine prescrite » } \\
(441)\end{array}$ \\
\hline $\begin{array}{l}\text { Kunac and Reith } \\
(2005)^{26}\end{array}$ & Nouvelle-Zélande & $\mathrm{PH}+\mathrm{UC}$ & 6 & 72 & NA & $\begin{array}{l}\text { "Manque de } \\
\text { formation sur } \\
\text { l'innocuité des } \\
\text { médicaments » (273) }\end{array}$ \\
\hline Lago et al. $(2012)^{27}$ & Italie & $\mathrm{PH}+\mathrm{UC}$ & 6 & 204 & $\begin{array}{l}\text { Mesures correctives } \\
\text { diverses (réel) }\end{array}$ & $\begin{array}{l}\text { "Prescription aux } \\
\text { SIP » (IC sommatif } \\
\text { de l'étape : } 420 \text { pré, } \\
140 \text { post) }\end{array}$ \\
\hline $\begin{array}{l}\text { Manrique-Rodríguez } \\
\text { et al. }(2014)^{28}\end{array}$ & Espagne & UC & 3 & 19 & $\begin{array}{l}\text { Automatisation des } \\
\text { procédures de pompes } \\
\text { intelligentes (réel) }\end{array}$ & $\begin{array}{l}\text { « Non-respect des } \\
\text { concentrations et } \\
\text { des heures } \\
\text { d'administration } \\
\text { standardisées en } \\
\text { infusions } \\
\text { intermittentes » } \\
\text { (210 pré, } 70 \text { post) }\end{array}$ \\
\hline Nguyen et al. (2013) & Canada & UC & 5 & 53 & NA & $\begin{array}{l}\text { "Pas de conseils aux } \\
\text { patients " } \\
\text { "Absence de } \\
\text { mention écrite } \\
\text { du conseil » (551) } \\
\end{array}$ \\
\hline
\end{tabular}


Tableau 1 (partie 2 de 2). Profil synthèse des AMDE et des AMDEC utilisées dans le circuit du médicament

\begin{tabular}{|c|c|c|}
\hline Référence & Pays & $\begin{array}{l}\text { Processus en } \\
\text { pharmacie (PH) } \\
\text { ou en unités de } \\
\text { soins, de cliniques } \\
\text { externes (UC) }\end{array}$ \\
\hline
\end{tabular}

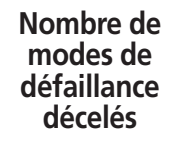

de

Interventions (protocole avec AMDE ou AMDEC pré-post seulement) et évaluation

post-implantation (implantation réelle ou simulée)

\begin{tabular}{llccr}
\hline $\begin{array}{l}\text { Nickerson et al. } \\
(2008)^{5}\end{array}$ & Canada & PH + UC & 4 & 78 \\
\hline Ofek et al. (2016) & Israël & UC & 5 & 13 \\
\hline Ponzetti et al. (2016) & Italie & PH + UC & ND & 12 \\
\hline $\begin{array}{l}\text { Robinson et al. } \\
(2006)^{32}\end{array}$ & États-Unis & PH + UC & 4 & \\
$\begin{array}{l}\text { Rodriguez-Gonzalez } \\
\text { et al. }(2015)^{33}\end{array}$ & Espagne & UC & 4 & 1 \\
$\begin{array}{l}\text { Saizy-Callaert et al. } \\
(2001)^{34}\end{array}$ & France & UC & 6 & \\
\hline
\end{tabular}

Shebl et al. $(2012)^{35} \quad$ Royaume-Uni $\quad$ PH + UC $\quad 4$

78

13

3

NA
$\begin{aligned} & \text { Passage de protocole IV } \\ & \text { vers SC (simulé) }\end{aligned}$

ND NA

$40 \quad$ Mesures correctives
diverses (réel)

11 NA

50

NA

Mode de
défaillance
comportant IC
le plus élevé
(valeur de l'IC
rapportée dans
l'étude)
ND

«Solution prédiluée contre-indiquée » (600)

ND

ND

(n)

\section{" Dose incorrecte »} (320 pré, 224 post)

«Modification de la prescription non détectée » (36) donné à l'heure prévue » « Médicament administré incorrectement » (576)

\begin{tabular}{|c|c|c|c|c|c|c|}
\hline $\begin{array}{l}\text { Silva and Cassiani } \\
(2013)^{36}\end{array}$ & Brésil & UC & 5 & 52 & $\begin{array}{l}\text { Mesures correctives } \\
\text { diverses (simulé) }\end{array}$ & ND \\
\hline $\begin{array}{l}\text { Arenas Villafranca } \\
\text { et al. }(2014)^{37}\end{array}$ & Espagne & $\mathrm{PH}+\mathrm{UC}$ & 2 & 82 & NA & $\begin{array}{l}\text { " Identification } \\
\text { erronée de la } \\
\text { prescription » (479) }\end{array}$ \\
\hline Walsh et al. $(2013)^{38}$ & États-Unis & UC & ND & 69 & NA & $\begin{array}{l}\text { "Erreur de } \\
\text { communication pour } \\
\text { changements de } \\
\text { dose » }\end{array}$ \\
\hline $\begin{array}{l}\text { Weingart et al. } \\
(2011)^{39}\end{array}$ & États-Unis & $\mathrm{PH}+\mathrm{UC}$ & 6 & 199 & NA & $\begin{array}{l}\text { « Non-respect de la } \\
\text { posologie par le } \\
\text { patient » }\end{array}$ \\
\hline $\begin{array}{l}\text { Wetterneck et al. } \\
(2009)^{40}\end{array}$ & États-Unis & UC & 8 & Plus de 200 & NA & ND \\
\hline $\begin{array}{l}\text { Williams and Talley } \\
(1994)^{41}\end{array}$ & États-Unis & $\mathrm{PH}+\mathrm{UC}$ & 6 & 26 & NA & $\begin{array}{l}\text { " Disponibilités de } \\
\text { doses létales » (576) }\end{array}$ \\
\hline $\begin{array}{l}\text { Yousefinezhadi } \\
\text { et al. }(2016)^{42}\end{array}$ & Iran & UC & 6 & 378 & NA & $\begin{array}{l}\text { " Mauvaise dose } \\
\text { administrée et durée } \\
\text { d'administration » } \\
(245)\end{array}$ \\
\hline \multicolumn{7}{|l|}{ Profil synthèse } \\
\hline 32 études & 14 pays & $\begin{array}{l}\mathrm{PH}(n=18) \\
\mathrm{UC}(n=30)\end{array}$ & $\begin{array}{l}\text { Min. : } 2 \\
\text { Max. : } 8\end{array}$ & $\begin{array}{c}\text { Min. : } 4 \\
\text { Max. : } 378\end{array}$ & ions $(n=10)$ & $\begin{array}{l}\text { Min. : } 7 \\
\text { Max. : } 729\end{array}$ \\
\hline
\end{tabular}

$\overline{\mathrm{AMDE}}$ = analyse des modes de défaillance et de leurs effets; $\mathrm{AMDEC}$ = analyse des modes de défaillance, de leurs effets et de leur criticité; $\mathrm{IC}=$ indice de criticité; Max. = maximum; Min. = minimum; $\mathrm{NA}=$ non applicable; $\mathrm{ND}=$ non déterminé; SIP = soins intensifs pédiatriques.

Le circuit du médicament en établissement de santé est complexe et comporte au moins 54 étapes $^{50}$, de la sélection des médicaments à leur élimination, en passant notamment par la prescription, la validation pharmaceutique, la préparation, l'administration et la consignation des renseignements au dossier du patient ou d'autres registres. Bien que des investissements importants aient été effectués dans ce circuit au cours de la dernière décennie, avec une informatisation de plusieurs processus et une robotisation de plusieurs de ces étapes (p. ex. ensacheuse, robot, pompe de remplissage, armoire automatisée, lecteur code-barres), force est de constater les nombreux modes de défaillance et les risques inhérents à cette complexité. À titre d'exemple, le rapport 2016-2017 sur les incidents et les accidents survenus lors de la prestation de soins de santé et de services sociaux au Québec révèle un total de 503447 incidents et accidents rapportés au cours de la dernière année. De ce nombre, ceux liés à la médication représentent $26,6 \%(n=133850)$ tandis que ceux liés à des équipements représentent $1,4 \%(n=7220)^{51}$. 


\section{Tableau 2. Profil synthèse des AMDE et des AMDEC utilisées dans le circuit du médicament}

\begin{tabular}{|c|c|c|c|c|c|c|}
\hline Référence & Pays & $\begin{array}{l}\text { Processus en } \\
\text { pharmacie (PH) } \\
\text { ou en unités de } \\
\text { soins, cliniques } \\
\text { externes (UC) }\end{array}$ & $\begin{array}{l}\text { Nombre de } \\
\text { professions } \\
\text { représentées } \\
\text { dans l'équipe } \\
\text { de recherche }\end{array}$ & $\begin{array}{l}\text { Nombre de } \\
\text { modes de } \\
\text { défaillance } \\
\text { détectés }\end{array}$ & $\begin{array}{l}\text { Interventions (protocole } \\
\text { avec AMDE ou AMDEC } \\
\text { pré-post seulement) }\end{array}$ & $\begin{array}{c}\text { Mode de } \\
\text { défaillance } \\
\text { comportant IC } \\
\text { le plus élevé } \\
\text { (valeur de l'IC } \\
\text { rapportée dans } \\
\text { l'étude) }\end{array}$ \\
\hline $\begin{array}{l}\text { Ashley and Armitage } \\
(2010)^{43}\end{array}$ & Royaume-Uni & $\mathrm{PH}+\mathrm{UC}$ & 3 & 30 & $\mathrm{NA}$ & $\begin{array}{l}\text { "Réaction } \\
\text { allergique » (16) }\end{array}$ \\
\hline Day et al. (2006) & États-Unis & UC & Au moins 3 & 21 & NA & $\begin{array}{l}\text { "Prescription } \\
\text { d'hémodialyse » (16) }\end{array}$ \\
\hline Esmail et al. $(2005)^{45}$ & Canada & UC & 5 & 13 & NA & $\begin{array}{l}\text { "L'infirmière prend } \\
\text { le mauvais } \\
\text { médicament » (12) }\end{array}$ \\
\hline Li et al. (2017) ${ }^{46}$ & Chine & $\mathrm{PH}+\mathrm{UC}$ & 6 & 5 & NA & $\begin{array}{l}\text { « Erreur de } \\
\text { prescription (dose, } \\
\text { soluté, fréquence, } \\
\text { horaire) » (16) }\end{array}$ \\
\hline $\begin{array}{l}\text { Ouellette-Piazzo } \\
\text { et al. }(2007)^{47}\end{array}$ & États-Unis & UC & $\mathrm{ND}$ & 20 & NA & Non spécifié \\
\hline $\begin{array}{l}\text { van Tilburg et al. } \\
(2006)^{48}\end{array}$ & Pays-Bas & $\mathrm{PH}+\mathrm{UC}$ & 7 & 61 & NA & $\begin{array}{l}\text { "Changement de la } \\
\text { prescription pas } \\
\text { relevé par la } \\
\text { pharmacie » } \\
\text { "Ancienne dose } \\
\text { servie par la } \\
\text { pharmacie après un } \\
\text { changement de la } \\
\text { prescription » (8) }\end{array}$ \\
\hline $\begin{array}{l}\text { Vélez-Díaz-Pallarés } \\
\text { et al. }(2013)^{49}\end{array}$ & Espagne & $\mathrm{PH}+\mathrm{UC}$ & 5 & 11 & NA & $\begin{array}{l}\text { "Erreurs de } \\
\text { prescription » (16) }\end{array}$ \\
\hline \multicolumn{7}{|l|}{ Profil synthèse } \\
\hline 7 études & Six pays & $\begin{array}{l}\mathrm{PH}(n=4) \\
\mathrm{UC}(n=7)\end{array}$ & $\begin{array}{l}\text { Min. : } 3 \\
\text { Max. : } 7\end{array}$ & $\begin{array}{l}\text { Min. : } 5 \\
\text { Max. : } 61\end{array}$ & Aucune intervention & $\begin{array}{l}\text { Min. : } 8 \\
\text { Max. : } 16\end{array}$ \\
\hline
\end{tabular}

$\overline{\mathrm{AMDE}}=$ analyse des modes de défaillance et de leurs effets; $\mathrm{AMDEC}$ = analyse des modes de défaillance, de leurs effets et de leur criticité; $\mathrm{IC}$ = indice de criticité; Max. = maximum; Min. = minimum; NA = non applicable; ND = non déterminé.

Ainsi, au moins $28 \%$ des incidents et accidents rapportés en établissement de santé au Québec sont liés au circuit du médicament. En tenant compte de la classification proposée par le National Coordinating Council for Medication Error Reporting and Prevention (NCCMERP), on compte parmi les événements indésirables liés à la médication, huit de catégorie I (associés à un décès), 11 de catégorie $\mathrm{H}$ (associés à des interventions de maintien de la vie), sept de catégories $\mathrm{G}$ (associés à des conséquences permanentes pour le patient) et 99 de catégories $\mathrm{F}$ (associés à des conséquences temporaires pour le patient). L'utilisation d'AMDE / AMDEC / AMDECS peut contribuer à l'analyse du processus et des étapes du circuit du médicament et mener ainsi à des interventions plus ciblées concernant le circuit du médicament. Ces analyses devraient être plus largement utilisées dans le réseau de la santé canadien.

Les AMDE / AMDEC / AMDECS incluses dans notre revue documentaire atteignent généralement les objectifs fixés, soit d'établir un inventaire des modes de défaillance potentiels associés à un processus et d'évaluer les conséquences potentielles de la survenue de ces défaillances. Seule 10 des 39 analyses retenues décrivent une démarche de type pré et postimplantation. En fait, quatre de ces 10 analyses présentent une méthode où l'équipe multidisciplinaire a été invitée à déterminer et à coter théoriquement des mesures correctives pré et postimplantation avant leur application éventuelle : les modes de défaillance, leurs effets et leur criticité. En apportant des changements à une pratique, on peut introduire de nouveaux modes de défaillance, ce qui rend la somme des IC difficilement interprétable. Une analyse pré et postimplantation revêt de l'intérêt surtout si l'on compare les indices de criticité avant et après le changement, plutôt que la seule comparaison de la somme des indices.

Même si les analyses AMDE / AMDEC / AMDECS n'atteignent leur plein potentiel de réduction des risques qu'en étant réalisées de façon pré et postimplantation, les analyses ponctuelles demeurent les plus fréquentes. Ce compromis en soi révèle combien la réalisation des AMDE / AMDEC / AMDECS est un exercice qui peut être exigeant. La réalisation de ce type d'analyse requiert des travaux préliminaires afin de sélectionner le processus et les étapes ciblées, de cartographier ces derniers et de revoir la littérature. De plus, l'analyse exige la mobilisation de divers professionnels durant plusieurs rencontres afin d'expliquer la méthode, de présenter la cartographie préliminaire des processus et des étapes, de déterminer les modes de défaillance et de procéder à la cotation de chacun des modes. La réalisation d'un AMDE / 
AMDEC / AMDECS peut s'échelonner sur plusieurs semaines et ne donne de bons résultats que lorsque tous les intervenants sont présents à toutes les rencontres. En outre, la nature de l'exercice demeure qualitative. La méthode permet de structurer des rencontres et d'encourager les échanges entre les membres d'une équipe multidisciplinaire sur la nature des modes de défaillance. Un tel effort d'analyse n'est pas forcément facile à répéter après un changement de pratique ou après l'implantation de mesures correctives au sein d'un établissement. Compte tenu de l'investissement personnel des participants dans la démarche, toute évaluation postérieure devrait préférablement reposer sur les mêmes panélistes. Toutefois, il est probablement plus judicieux de faire une étude observationnelle avec un protocole spécifique à la détermination de l'impact des changements mis en place. Par conséquent, la méthode AMDE ne peut pas avoir d'impact direct, car elle n'est conçue que pour déterminer les situations problématiques.

Même dans les publications médicales plus récentes, les articles retenus employant la méthode AMDECS demeurent minoritaires par rapport à ceux portant sur l'AMDE et l'AMDEC, malgré le fait que l'AMDECS relève directement du domaine de la santé. Rah et collab. ${ }^{52}$ ont comparé l'AMDEC et l'AMDECS pour déceler les processus associés à un risque élevé en chirurgie. Bien que les résultats concordent à $85 \%$ entre les deux approches, les auteurs soulignent l'importance d'essayer différentes approches et d'utiliser celle qui correspond davantage à ses besoins ${ }^{52}$.

La consultation des 39 études met en évidence une grande hétérogénéité, tant pour les processus et les étapes ciblés que pour les modes de défaillance détectés et les cotes attribuées à chacun. Cette hétérogénéité est une conséquence de l'application de la méthode AMDE / AMDEC / AMDECS. Chaque établissement, chaque équipe multidisciplinaire, chaque processus comporte ses particularités. Il faut très souvent ajuster les échelles de cotation afin d'obtenir un degré suffisant de résolution pour commenter le processus étudié. Par exemple, si on analyse une étape qui se produit 1000 fois par jour avec un taux d'erreur de $1 \%$, il faut probablement faire ajuster la cote de fréquence de survenue d'un mode de défaillance par une échelle utilisant des heures et des jours plutôt qu'une échelle type utilisant davantage des joursmois-années. En outre, la cotation des modes de défaillance dépend de la connaissance fine des processus et des étapes qu'ont les panélistes, mais également des données locales et de la littérature qui aident à baliser la fréquence, la sévérité et la capacité de détection des modes de défaillance.

En somme, les résultats d'une AMDE / AMDEC / AMDECS sont avant tout utiles pour un établissement donné. Ils lui servent à prendre conscience des modes de défaillance, de leur hiérarchie et à encourager la mise en place de mesures correctives. Si l'on envisage de répéter cette méthode dans le temps, afin de se comparer à soi-même, la comparaison doit se faire à l'aide d'un échantillon comparable de modes de défaillance ou en étant conscient de l'introduction de nouveaux modes. S'il est peu utile de comparer les indices globaux de criticité entre les études ou entre des établissements, il est intéressant de consulter les différents modes de défaillance d'un autre établissement et la magnitude du risque associée à chacun, peu importe si on réalise ou non de telles analyses localement.

Cette revue documentaire comporte des limites. Tout d'abord, notre étude n'a eu recours qu'à une seule base de données. D'autres sources de données pourraient donc être envisagées (p. ex. Embase, Google Scholar) afin de découvrir des études supplémentaires. Ensuite, notre étude présente uniquement un profil descriptif général. Enfin, une analyse qualitative détaillée de chacune des études pourrait permettre de déterminer les difficultés rencontrées, les meilleures échelles de cotations et les approches de rencontres optimales.

\section{CONCLUSION}

Cette revue de littérature porte sur 39 articles publiés ayant utilisé l'approche AMDE / AMDEC / AMDECS dans le cadre du circuit du médicament. La plupart des études utilisent l'approche AMDE / AMDEC, tandis que l'AMDECS n'est que rarement employée. Il existe en outre une grande hétérogénéité entre les différentes analyses sur le plan des processus ciblés et des méthodes employées. Seule une minorité des études font état d'analyses réalisées avant et après l'implantation de mesures correctives. L'AMDE / AMDEC / AMDECS est une approche multidisciplinaire très utile pour cartographier un processus de soins, déterminer les modes de défaillance et prioriser les actions correctives. Il faudrait encourager son usage pour l'optimisation du circuit du médicament.

\section{Références}

1. Analyse des modes de défaillance, de leurs effets et de leur criticité. Dans : Le grand dictionnaire terminologique (GDT). Montréal (QC) : Office québécois de la langue française; 2005. Publié au : www.granddictionnaire.com/fiche Oqlf.aspx?Id_Fiche=2068801. Consulté le 28 décembre 2017.

2. Duwe B, Fuchs BD, Hansen-Flaschen J. Failure mode and effects analysis application to critical care medicine. Crit Care Clin. 2005;21(1):21-30.

3. Failure mode and effects analysis in health care: proactive risk reduction. $2^{\mathrm{e}}$ édition. Oak Brook (IL) : Joint Commission Resources Inc; 2005.

4. Landy G. AMDEC guide pratique. $2^{\mathrm{e}}$ édition. La Plaine Saint-Denis (France) : AFNOR; 2007.

5. Nickerson T, Jenkins M, Greenall J. Using ISMP Canada’s framework for failure mode and effects analysis: a tale of two FMEAs. Healthc Q. 2008;11 (3 Spec No.):40-6.

6. Liu HC. FMEA using uncertainty theories and MCDM methods. Singapore : Springer Science+Business Media Singapore; 2016.

7. DeRosier J, Stalhandske E, Bagian JP, Nudell T. Using health care failure mode and effect analysis: the VA National Center for Patient Safety's prospective risk analysis system. Jt Comm J Qual Improve. 2002;28(5):248-67.

8. Patient safety systems (PS). Dans : Comprehensive accreditation manual for hospitals. Mis-à-jour no 2. Oak Brook (IL) : The Joint Commission; 2016. Publié au : https://www.jointcommission.org/assets/1/18/PSC_for_Web.pdf. Consulté le 28 décembre 2017.

9. Human factors analysis in patient safety systems. The Source [publication du Joint Commission]. 2015;13(4):1,7-10. Publié au : https://www. jointcommission.org/assets/1/6/HumanFactorsThe_Source.pdf. Consulté le 28 décembre 2017. 
10. VA National Center for Patient Safety. Healthcare failure mode and effect analysis (HFMEA). Washington (DC) : US Department of Veterans Affairs; 2015. Publié au : https://www.patientsafety.va.gov/professionals/onthejob/ hfmea.asp. Consulté le 28 décembre 2017.

11. Pratiques organisationnelles requises livret $2017.2^{\mathrm{e}}$ version. Ottawa $(\mathrm{ON})$ : Agrément Canada; 2017. En vente au : https://store.accreditation.ca/ products/pratiques-organisationnelles-requises-livret-2017-2e-versionjanvier-2017. Consulté le 28 décembre 2017.

12. Adachi W, Lodolce AE. Use of failure mode and effects analysis in improving the safety of i.v. drug administration. Am J Health Syst Pharm. 2005; 62(9):917-20.

13. Apkon M, Leonard J, Probst L, DeLizio L, Vitale R. Design of a safer approach to intravenous drug infusions: failure mode effects analysis. Qual Saf Health Care. 2004;13(4):265-71.

14. Armitage G, Hodgson I, Wright J, Bailey K, Mkhwana E. Exploring the delivery of antiretroviral therapy for symptomatic HIV in Swaziland: threats to the successful treatment and safety of outpatients attending regional and district clinics. BMJ Qual Saf. 2011;20(1):52-9.

15. Baker DK, Hoffman JM, Hale GA, Spunt SL, Sanderlin D, Rodman JH, et al. Analysis of patient safety: converting complex pediatric chemotherapy ordering processes from paper to electronic systems. Dans : Henriksen K, Battles JB, Keyes MA, Grady ML, rédacteurs. Advances in patient safety: new directions and alternative approaches. Vol. 2: Culture and redesign. Rockville (MD) : Agency for Healthcare Research and Quality (US); 2008.

16. Berruyer M, Atkinson S, Lebel D, Bussières JF. Utilisation de l'insuline en établissement de santé : une analyse des modes de défaillance. Pharmactuel. 2014;47:55-61.

17. Bonnabry P, Cingria L, Sadeghipour F, Ing H, Fonzo-Christe C, Pfister RE. Use of a systematic risk analysis method to improve safety in the production of paediatric parenteral nutrition solutions. Qual Saf Health Care. 2005; 14(2):93-8.

18. Bonnabry P, Cingria L, Ackermann M, Sadeghipour F, Bigler L, Mach N. Use of a prospective risk analysis method to improve the safety of the cancer chemotherapy process. Int J Qual Health Care. 2006;18(1):9-16.

19. Bonnabry P, Despont-Gros C, Grauser D, Casez P, Despond M, Pugin D, et al. A risk analysis method to evaluate the impact of a computerized provider order entry system on patient safety. J Am Med Inform Assoc. 2008;15(4): 453-60.

20. Coles G, Fuller B, Nordquist K, Kongslie A. Using failure mode effects and criticality analysis for high-risk processes at three community hospitals. Jt Comm J Qual Patient Saf. 2005;31(3):132-40.

21. de la Riva P, Martinez Zabaleta MT, Arruti González M, Urtasun Ocariz MA. Failure mode and effect analysis applied to the procedure for intrathecal chemotherapy. Neurologia. 2015;30(1):62-4.

22. Delage E, Tourel J, Martin B, Guérin A, Moussa A, Lacroix A, et al. Perfusions continues et pompes intelligentes en néonatologie : une analyse " pré-post " des modes de défaillance, de leurs effets et de leur criticité (AMDEC). Can J Hosp Pharm. 2015;68(5):406-11.

23. Dräger $S$. Who is responsible for a safe discharge from hospital? A prospective risk analysis in the German setting. Z Evid Fortbild Qual Gesundhwes. 2016;113:9-18.

24. Hosoya K, Mochinaga S, Emoto A, Yokoo H, Tokushima H, Egoshi M, et al. Failure mode and effects analysis of medication adherence in patients with chronic myeloid leukemia. Int J Clin Oncol. 2015;20(6):1203-10.

25. Kaestli LZ, Cingria L, Fonzo-Christe C, Bonnabry P. Prospective risk analysis and incident reporting for better pharmaceutical care at paediatric hospital discharge. Int J Clin Pharm. 2014;36(5):953-62.

26. Kunac DL, Reith DM. Identification of priorities for medication safety in neonatal intensive care. Drug Saf. 2005;28(3):251-61.

27. Lago P, Bizzarri G, Scalzotto F, Parpaiola A, Amigoni A, Putoto G, et al. Use of FMEA analysis to reduce risk of errors in prescribing and administering drugs in paediatric wards: a quality improvement report. BMJ Open. 2012; 2:e001249.

28. Manrique-Rodríguez S, Sánchez-Galindo AC, López-Herce J, CallejaHernández MÁ, Iglesias-Peinado I, Carrillo-Álvarez A, et al. Risks in the implementation and use of smart pumps in a pediatric intensive care unit: application of the failure mode and effects analysis. Int J Technol Assess Health Care. 2014;30(2):210-7.
29. Nguyen C, Côté J, Lebel D, Caron E, Genest C, Mallet M, et al. The AMÉLIE project: failure mode, effects and criticality analysis: a model to evaluate the nurse medication administration process on the floor. $J$ Eval Clin Pract. 2013;19(1):192-9.

30. Ofek F, Magnezi R, Kurzweil Y, Gazit I, Berkovitch S, Tal O. Introducing a change in hospital policy using FMEA methodology as a tool to reduce patient hazards. Isr J Health Policy Res. 2016;5:30.

31. Ponzetti C, Canciani M, Farina M, Era S, Walzer S. Administrative risk quantification of subcutaneous and intravenous therapies in Italian centers utilizing the failure mode and effects analysis approach. Clinicoecon Outcomes Res. 2016;8:353-9.

32. Robinson DL, Heigham M, Clark J. Using failure mode and effects analysis for safe administration of chemotherapy to hospitalized children with cancer. Jt Comm J Qual Patient Saf. 2006;32(3):161-6.

33. Rodriguez-Gonzalez CG, Martin-Barbero ML, Herranz-Alonso A, DurangoLimarquez MI, Hernandez-Sampelayo P, Sanjurjo-Saez M; iPharma. Use of failure mode, effect and criticality analysis to improve safety in the medication administration process. J Eval Clin Pract. 2015;21(4):549-59.

34. Saizy-Callaert S, Causse R, Thébault A, Chouaïd C. Analysis of mode of failure, their effects and criticality: improving of the hospital drug prescribing process. Therapie. 2001;56(5):525-31.

35. Shebl NA, Franklin BD, Barber N. Failure mode and effects analysis outputs: are they valid? BMC Health Serv Res. 2012;12:150.

36. Silva AEBC, Cassiani SHB. Prospective risk analysis of the anti-infective medication administration process. Rev Lat Am Enfermagem. 2013;21(Spec No.):233-41.

37. Arenas Villafranca JJ, Gómez Sánchez A, Nieto Guindo M, Faus Felipe V. Using failure mode and effects analysis to improve the safety of neonatal parenteral nutrition. Am J Health Syst Pharm. 2014;71(14):1210-8.

38. Walsh KE, Mazor KM, Roblin D, Biggins C, Wagner JL, Houlahan K, et al. Multisite parent-centered risk assessment to reduce pediatric oral chemotherapy errors. J Oncol Pract. 2013;9(1):e1-7.

39. Weingart SN, Spencer J, Buia S, Duncombe D, Singh P, Gadkari M, et al. Medication safety of five oral chemotherapies: a proactive risk assessment. J Oncol Pract. 2011;7(1):2-6.

40. Wetterneck TB, Hundt AS, Carayon P. FMEA team performance in health care: a qualitative analysis of team member perceptions. J Patient Saf. 2009; 5(2):102-8.

41. Williams E, Talley R. The use of failure mode effect and criticality analysis in a medication error subcommittee. Hosp Pharm. 1994;29(4): 331-2,334-6,339.

42. Yousefinezhadi T, Jannesar Nobari FA, Behzadi Goodari F, Arab M. A case study on improving intensive care unit (ICU) services reliability: by using process failure mode and effects analysis (PFMEA). Glob J Health Sci. 2016; 8(9):207-33

43. Ashley L, Armitage G. Failure mode and effects analysis: an empirical comparison of failure mode scoring procedures. J Patient Saf. 2010;6(4):210-5.

44. Day S, Dalto J, Fox J, Turpin M. Failure mode and effects analysis as a performance improvement tool in trauma. J Trauma Nurs. 2006;13(3):111-7.

45. Esmail R, Cummings C, Dersch D, Duchscherer G, Glowa J, Liggett G, et al.; Patient Safety and Adverse Events Team. Using healthcare failure mode and effect analysis tool to review the process of ordering and administrating potassium chloride and potassium phosphate. Healthc Q. 2005;8(Spec No.):73-80.

46. Li G, Xu B, He RX, Zhang SX. Using healthcare failure mode and effect analysis to reduce intravenous chemotherapy errors in Chinese hospitalized patients. Cancer Nurs. 2017;40(2):88-93.

47. Ouellette-Piazzo K, Asfaw B, Cowen J. CT healthcare failure mode effect analysis (HFMEA): the misadministration of IV contrast in outpatients. Radiol Manage. 2007;29(1):36-44; quiz 45-7.

48. Van Tilburg CM, Leistikow IP, Rademaker CM, Bierings MB, van Dijk AT. Health care failure mode and effect analysis: a useful proactive risk analysis in a pediatric oncology ward. Qual Saf Health Care. 2006;15(1):58-63.

49. Vélez-Díaz-Pallarés M, Delgado-Silveira E, Carretero-Accame ME, BermejoVicedo T. Using healthcare failure mode and effect analysis to reduce medication errors in the process of drug prescription, validation and dispensing in hospitalised patients. BMJ Qual Saf. 2013;22(1):42-52. 
50. Jodoin J, Lantin S, Bussières JF, Bouchard S, Cohen E, Lussier-Labelle F, et al. Les systèmes automatisés et robotisés utilisés pour la distribution des médicaments dans les établissements de santé au Québec. Rapport et recommandations du groupe de travail. Québec (QC) : Ministère de la santé et des services sociaux; 2005.

51. Lavoie D, rédactrice. Rapport 2016-2017 sur les incidents et les accidents survenus lors de la prestation de soins de santé et de services sociaux au Québec. Québec (QC) : Ministère de la santé et des services sociaux; 2017. Publié au : http://publications.msss.gouv.qc.ca/msss/fichiers/2017/17-735-01W.pdf. Consulté le 28 décembre 2017.

52. Rah JE, Manger RP, Yock AD, Kim GY. A comparison of two prospective risk analysis methods: traditional FMEA and a modified healthcare FMEA. Med Phys. 2016;43(12):6347.

Émile Demers, Pharm. D., M. Sc., est résident en pharmacie, Unité de recherche en pratique pharmaceutique, Centre hospitalier universitaire Sainte-Justine, Montréal, Québec.

Laurence Collin-Lévesque, Pharm. D., M. Sc., est résident en pharmacie, Unité de recherche en pratique pharmaceutique, Centre hospitalier universitaire Sainte-Justine, Montréal, Québec.

Marianne Boulé, Pharm. D., M. Sc., est résidente en pharmacie, Unité de recherche en pratique pharmaceutique, Centre hospitalier universitaire Sainte-Justine, Montréal, Québec.
Sophie Lachapelle, Pharm. D., M. Sc., est résidente en pharmacie, Unité de recherche en pratique pharmaceutique, Centre hospitalier universitaire Sainte-Justine, Montréal, Québec.

Christina Nguyen, B. Sc., M. Sc., est pharmacienne, Unité de recherche en pratique pharmaceutique et département de pharmacie, Centre hospitalier universitaire Sainte-Justine, Montréal, Québec.

Denis Lebel, B. Pharm., M. Sc., FCSHP, est Adjoint au chef, Unité de recherche en pratique pharmaceutique et département de pharmacie, Centre hospitalier universitaire Sainte-Justine, Montréal, Québec.

Jean-François Bussières, B. Pharm., M. Sc., M. B. A., FCSHP, FOPQ, est Chef, Unité de recherche en pratique pharmaceutique et département de pharmacie, Centre hospitalier universitaire Sainte-Justine, et professeur titulaire de clinique, Faculté de pharmacie, Université de Montréal, Montréal, Québec.

Intérets concurrents: Aucun déclaré.

\section{Adresse de correspondance :}

Jean-François Bussières

Département de pharmacie

Centre hospitalier universitaire Sainte-Justine

3175, chemin de la côte Sainte-Catherine Montréal QC H3T 1C5

Courriel : jf.bussieres@ssss.gouv.qc.ca

Financement : Aucun reçu. 